\title{
Computerised and manual cephalometry used for accuracy and reliability of landmark identification
}

\author{
Jyoti Singh Gaharwar \\ Associate Professor, Dept. of Dentistry, Amaltas Institute of Medical Sciences, Dewas, Madhya Pradesh, India
}

*Corresponding Author: Jyoti Singh Gaharwar

Email: jyotisinghgh@yahoo.com

\begin{abstract}
Introduction: Digital imaging offers several potentials advantages over traditional radiography like reduced radiation eposure, elimination of chemical processing and image enhancement using various algorithms.

Materials and Methods: The study sample consisted of 25 lateral cephalometric radiographs which were selected randomly from the data files.

Results: The Result of computerised cephalometric tracing method by digitization is more reliable and consistent as compared to manual cephalometric tracing method.
\end{abstract}

Keywords: Digital cephalometry, Manual cephalometric tracings, Cephalometric data, Cephalometric analysis.

The cephalometric parameters were statistically analyzed by calculating their means and standard deviations i.e. descriptive statistics. Then the means of measurements obtained by manual cephalometric tracings were compared with means of computerized lateral cephalometric tracings with the help of student's unpaired 't' test.

The definitions and formulae for calculating the mean, standard deviation, and tests for significance are given below:-

\section{Mean}

It is defined as summing up all observations and dividing the total by the number of observations. It is calculated as,

$\bar{X}=\frac{\Sigma X}{n}$

Mean, $\mathrm{x}$

Where,

$\mathbf{X}=$ The value of the variables.

$\boldsymbol{\Sigma}=$ Sum of the values.

$\mathbf{n}=$ Number of observations.

\section{Standard deviation}

The standard deviation is the most frequently used measure of deviation. It is the most frequently used measure of deviation. It is defined as the root mean square deviation and is denoted by s or SD.

$$
\mathrm{s}=\sqrt{\frac{1}{N-1} \sum_{i=1}^{N}\left(x_{i}-\bar{x}\right)^{2}}
$$

Where,

$\mathbf{X}=$ mean

$\boldsymbol{\Sigma}=$ Sum of the values

$\mathbf{N}=$ Number of observations

\section{Standard error of mean}

Standard error of mean measures how precisely the population mean is estimated by the mean of the given sample. The standard error various with the size of the standard deviation. Greater the SD, greater the $\mathrm{SE}>$

$\mathrm{SE}=\frac{S}{\sqrt{n}}$

Where,

$\mathrm{S}=$ Standard deviation

$\mathrm{N}=$ Number of observations

\section{Student's "t-test"}

It is used to test whether the means of two independent samples are significantly different. It is denoted by " $t$ " The formula is

$$
\mathrm{t}=\frac{x_{1}-X_{2}^{-}}{s x_{1} x_{2}-\sqrt{\frac{2}{n}}}
$$

Where in,

$$
s_{x 1 \times 2}=\sqrt{\frac{1}{2}\left(s^{2} x_{1}+s^{2} x_{2}\right)}
$$

Where,

$\mathrm{S}_{\mathrm{x} 1 \times 2}=$ Combined standard deviation

$\mathrm{X}_{1}=$ Mean of the first Sample

$\mathrm{X}_{2}=$ Meant of the second sample

$\mathrm{n}_{1}=$ Sample size of the first sample

$\mathrm{n}_{2}=$ Sample size of the second sample

$\mathrm{s}_{\mathrm{x} 1}=$ Standard deviation of the first sample

$\mathrm{s}_{\mathrm{x} 2}=$ Standard deviation of the second sample

Digital Imaging offers several potential advantages over traditional radiography like reduced radiation exposure, elimination of chemical processing and image enhancement. It also allows automated cephalometric analysis, soft tissue 
superimposition/morphing, archiving and transmission of the digital images. Digital radiography gives us the advantage of enhancing the diagnostic quality of digital images using various algorithms. ${ }^{2}$

Studies have shown that images captured from flatbed scanner are reliable as compared to their corresponding analogue headfilms for use in clinical practice, but cannot be used for research products. Many factors affect the quality of the scanned image like dpi value, number of piels and amount of illumination, which have a direct effect on the landmark identification. Thus a standardition of the scanning setup is mandatory to advice a good quality image. ${ }^{3}$

\section{Discussion}

Many Computer systems were developed in the following years with an aim to simplify the process of cephalometric analysis. It is found that Manual tracing was less precise than digital tracing. Digital cephalometric analysis not only saves time but also makes the landmark identification more precise by removing the errors caused during measurement using ruler and protector. Additionally, digital image can be manipulated to process the image and alter its visual appearance which can facilitate landmark identification.

Table 1: Mean, minimum, maximum \& standard deviation of various parameters in Steiner's Analysis

\begin{tabular}{|c|l|c|c|c|c|c|c|c|c|}
\hline Sl. No. & \multicolumn{1}{|c|}{ Parameter } & \multicolumn{2}{c|}{ Mean } & \multicolumn{2}{c|}{ Minimum } & \multicolumn{2}{c|}{ Maximum } & \multicolumn{2}{c|}{ Standard Deviation } \\
\hline & & $\begin{array}{c}\text { Group } \\
\text { A }\end{array}$ & $\begin{array}{c}\text { Group } \\
\text { B }\end{array}$ & $\begin{array}{c}\text { Group } \\
\text { A }\end{array}$ & $\begin{array}{c}\text { Group } \\
\text { B }\end{array}$ & $\begin{array}{c}\text { Group } \\
\text { A }\end{array}$ & $\begin{array}{c}\text { Group } \\
\text { B }\end{array}$ & $\begin{array}{c}\text { Group } \\
\text { A }\end{array}$ & $\begin{array}{c}\text { Group B } \\
\end{array}$ \\
\hline 1 & SNA Angle (degree) & 81.9 & 82.2 & 72.1 & 72.9 & 90.3 & 91.3 & 8.2 & 7.9 \\
\hline 2 & SNB Angle (degree) & 77.8 & 78.5 & 70.4 & 71.4 & 88.4 & 89.3 & 6.9 & 7.1 \\
\hline 3 & ANB Angle (degree) & 3.6 & 4.1 & -2.1 & -2.6 & 6.8 & 7.1 & 3.1 & 3.8 \\
\hline 4 & GoGn - SN Angle (degree) & 31.2 & 30.8 & 27.4 & 26.4 & 40.2 & 41.3 & 8.7 & 8.3 \\
\hline 5 & U1 - NA angular (degree) & 24.7 & 23.4 & 18.3 & 17.7 & 36.1 & 37.1 & 5.1 & 5.3 \\
\hline 6 & U1 - NA linear (mm) & 6.2 & 6.1 & 3.7 & 4.1 & 8.5 & 8.8 & 3.4 & 3.6 \\
\hline 7 & L1 - NB angular (degree) & 23.9 & 22.7 & 17.9 & 17.3 & 32.5 & 33.2 & 5.6 & 5.9 \\
\hline 8 & L1 - NB linear (mm) & 5.3 & 5.9 & 3.6 & 3.4 & 7.1 & 7.4 & 2.9 & 3.1 \\
\hline 9 & Interincisal angle (degree) & 124.5 & 125.7 & 111.6 & 112.5 & 145.8 & 146.2 & 11.8 & 12.2 \\
\hline 10 & Occlusal - SN (angular) & 14.1 & 14.9 & 11.5 & 12.1 & 19.2 & 19.8 & 3.8 & 4.1 \\
\hline
\end{tabular}

Key:

Group A: Manual cephalometric tracing group

Group B: Computerized cephalometric tracing group (B)

Table 2: "t" values for various parameters between Group A and Group B

\begin{tabular}{|c|c|c|c|c|}
\hline S. No. & Parameter & 't'” value & Probability & Significance \\
\hline 1 & SNA Angle (degree) & 0.74 & 0.51 & NS \\
\hline 2 & SNB Angle (degree) & 1.13 & 0.41 & NS \\
\hline 3 & ANB Angle (degree) & 0.64 & 0.471 & NS \\
\hline 4 & GoGn - SN Angle (degree) & 1.26 & 0.87 & NS \\
\hline 5 & U1 - NA angular (degree) & 0.34 & 0.72 & NS \\
\hline 6 & U1 - NA linear (mm) & 6.3 & 0.1 & HS \\
\hline 7 & L1 - NB angular (degree) & 1.52 & 0.272 & NS \\
\hline 8 & L1 - NB linear (mm) & 8.31 & 0.21 & HS \\
\hline 9 & Interincisal angle (degree) & 1.37 & 0.862 & NS \\
\hline 10 & Occlusal - SN (angular) & 3.24 & 0.001 & HS \\
\hline
\end{tabular}

Key:

NS: Not Significant

HS: Highly Significant

Table 3:

\begin{tabular}{|c|l|c|c|c|}
\hline S. No. & \multicolumn{1}{|c|}{ Parameter } & "t" value & Probability & Significance \\
\hline 1 & SNA Angle (degree) & 0.74 & 0.51 & NS \\
\hline 2 & SNB Angle (degree) & 1.13 & 0.41 & NS \\
\hline 3 & ANB Angle (degree) & 0.64 & 0.471 & NS \\
\hline 4 & GoGn - SN Angle (degree) & 1.26 & 0.87 & NS \\
\hline 5 & U1 - NA angular (degree) & 0.34 & 0.72 & NS \\
\hline 6 & U1 - NA linear (mm) & 6.3 & 0.1 & HS \\
\hline 7 & L1 - NB angular (degree) & 1.52 & 0.272 & NS \\
\hline 8 & L1 - NB linear (mm) & 8.31 & 0.21 & HS \\
\hline 9 & Interincisal angle (degree) & 1.37 & 0.862 & NS \\
\hline 10 & Occlusal - SN (angular) & 3.24 & 0.001 & HS \\
\hline
\end{tabular}




\section{Source of Funding}

None.

\section{Conflict of Interest}

None.

\section{References}

1. Broadbent BH. A new x-ray technique and its application to orthodontics. Angle Orthod. 1931;1(2):45-66.

2. Cohen AM. Uncertainty in cephalometrics. Br J Orthod. 1984;11(1):44-8.

3. Baumrind S, Frantz RC. The reliability of head film measurements 1. Landmark identification. Am J Orthod. 1980:45-65.

4. Rudolph DJ. Automatic computerized identification of cephalometric landmarks. Am J Orthod Dentofacial Orthop. 1998;113:173-9.

5. Forsyth DB, Shaw WC. Digital imaging of cephalometric radiography, part 1: Advantages and limitations of digital imaging. Angle Orthod. 1996;66:37-42.

6. Forsyth DB, Davis DN. Assessment of an automated cephalometric analysis system. Eur J Orthod. 1996;18:471-8.

7. Chen SK, Chen YJ, Yao CC, Chang HF. Enhanced speed and precision of Measurement in a computer-assisted digital cephalometric analysis system. Angle Orthod. 2004;74:501-7.

8. Macri V, Wenzel A. Reliability of landmark recording on film and digital lateral cephalograms. Eur J Orthod. 1993;15:13748.

9. Power G, Breckon J, Sherriff M, McDonald F. Dolphin Imaging Software: an analysis of the accuracy of cephalometric digitization and orthognathic prediction. Int $J$ Oral Maxillofac Surg. 2005;34:619-26.

10. Ongkosuwito EM, Katsaros C, Bodegom JC, Kuijpers-Jagtman AM. Digital cephalometrics. Ned Tijdschr Tandheelkd. 2004;111:266-70.

11. Bassignani MJ, Bubash-Faust L, Ciambotti J, Moran R, McIlhenny J. Conversion of teaching file cases from film to digital format: a comparison between use of a diagnosticquality digitizer and use of a flatbed scanner with transparency adapter. Acad Radiol. 2003;10:536-42.

12. Bruntz LQ, Palomo JM, Baden S, Hans MG. A comparison of scanned lateral cephalograms with corresponding original radiographs. Am J Orthod Dentofacial Orthop. 2006;130:340-6.

13. Chen YJ, Chen SK, Chang HF, Chen KC. Comparison of landmark identification in traditional versus computer-aided digital cephalometry. Angle Orthodontist. 2000;70:387-92.

14. Omur Polat-Ozsoy, Aylin Gokcelik and T. Ufuk Toygar Menkoglu. Differences in cephalometric measurements: a comparison of digital versus hand-tracing methods Eur J Orthod. 2009;29:105-8.

15. Turner PJ, Weerakone S. An evaluation of the reproducibility of landmark identification using scanned cephalometric images. J Orthod. 2001;28:221-9.

16. Shinawatra L, Anuwongnukroh L, Dechkunakorn S, Sinthanayothin C. Reliability of cephalometric measurements a comparison of digital image input techniques (scanner Vs digital camera) used for cephalometric analysis and its use in orthodontic treatment planning. Part - I. Am

17. Kula K, Kula JT, Glaros A, Barlow J. Evaluation of Printed and monitor-displayed images taken with digital cameras and imported into the dolphin imaging system. Semin Orthod. 2004;10:220-5.

18. Riedel RA. Cephalometrics \& its relation to orthodontic therapy. Am J Orthod. 1950;20:168-78.

19. Steiner CC. Cephalometrics for you and me. Am J Orthod. 1953;39:729-55.
20. Steiner CC. Cephalometrics as an aid to planning and assessing orthodontic Treatment. Am J Orthod. 1960;46:721-35.

21. Merrifield LL. The profile line as an evaluating facial esthetics. Am J Orthod. 1966;52:804-22.

22. Merrifield LL. Space analysis and differential diagnosis. Am J Orthod. 1978;01:715-29.

23. McDowell CS. A Reappraisal of Cephalometrics. J Clin Orthod. 1970;82-92.

24. Worms FW. Surgical orthodontic treatment planning: Profile analysis and mandibular surgery. Angle Orthod. 1976;46(1):125.

25. Holdaway RA. A soft tissue Cephalometric analysis and its use in orthodontic treatment planning - Part I Am J Orthod. 1983;84:1-28

26. Burstone CJ. The integumental profile. Am J Orthod 1958;44:1-25.

27. Ferrario VF. Head posture and cephalometric analyses. Am J Orthod Dentofac Orthop. 1994;106:257-64.

28. Raju SN, Prasad KG, Jayade VP. A modified approach for obtaining cephalograms in the natural head position. J Orthod. 2001;28(1):25-8.

29. Moorrees CFA. Natural head position - a revival. Am J Orthod Dentofac Orthop. 1994;105:512-3.

30. Lundstrom A, Lundstrom F. The Frankfort horizontal as a basis for cephalometric analysis. Am J Orthod Dentofac Orthop. 1995;107:537-40.

31. Bergman RT. Cephalometric soft tissue facial analysis. Am. $J$ Orthod Dentofac Orthop. 1999;116:373-89.

32. Arnett GW, Bergman RT. Facial keys to orthodontic Diagnosis \& Treatment planning. Part-II Am J Orthod. 1993;103:395411.

33. Matoula S and Pancherz H. Skeletofacial morphology of Attractive and Non attractive faces. Angle Orthod. 2006;76:204-10.

34. Taylor TS. Exposure reduction and image quality in orthodontic radiology - A review of literature. Am J Orthod Dentofac Orthop. 1988;93:68-77.

35. Cohen A.M. Uncertainity in cephalometrics. Br J Orthod. 1984;11:44-48.

36. Baumrind and Frantz R.C. The reliability of headfilm measurements- landmark identification. Am J Orthod. 1971;60(2):111-27.

37. Walker G. Cephalometric analysis of dentofacial normals; Am J Orthod Dentofac Orthop.1997;111(6):646-9.

38. Erik Eriksen and Beni Solow. Linearity of cephalometric digitizers. Eur J Orthod. 1991;13:337-42.

39. Luc PM. Tourne. Accuracy of commercially available digitizer: a new method for assessment of errors in linearity. Angle Orthod. 1996;66(6):325-40.

40. Chebib F.S. On line computer system for the analysis of cephalometric radiographs. Br J Orthod. 1979;46(4):305-11.

41. Houston WJB. The application of computer aided digital analysis to orthodontic records. Eur J Orthod. 1979;1:71-9.

42. Richard D. Faber. Computerized interactive orthodontic treatment planning. Am J Orthod. 1978;73(1):36-46

43. Alfredo de Alba YL. Effect of orthodontic intermaxillary Class III mechanics on craniofacial structures Part 2: Computerized cephalometrics: Br J Orthod. 1979;49(1):29-36.

44. Baumrind S, Miller DM. Computer-aided head film analysis: The university of California San Francisco method. Am J Orthod. 1980;78(1):41-65.

45. Olav Bondevik. The digital read-out system CM-1: An instrument for rationsl measuring on radiographic headplates and dental models. Eur J Orthod. 1981;3:1-8.

46. Konchak PA, Koelher JA. A Pascal computer program for digitizing lateral cephalometric radiographs. Am J Orthod. 1985; 14:197-200. 
47. Sandler PJ. Reproducibility of cephalometric measurements: Br J Orthod. 1998;15(7):105-10.

48. Brown T, Amanda HA. Computer-assisted location of reference points in three dimentions of radiographic cephalometry. Am J Orthod. 1989;95(6):490-8.

49. Chaconas SJ, Engel GA, Gianelly A, Nanda RS. The Digigraph Work Station Part- I - basic concepts. J Cl Orthod. 1990;24(1):360-7.

50. Alexander RG, Gorman JC, Gummons DC, Jacobson RL. The digiGraph work station part II: clinical management. $\mathrm{JCl}$ Orthod. 1990;24(2):402-7.

51. Mustafa YA, Nagwa Helmy EM, Salah A, Rasmy EM. Automated cephalometric soft tissue analysis. JCl Orthod. 1990;539-43.

52. Oliver RG. Cephalometric analysis comparing five different methods. Br J Orthod. 1991;18(4):277-83.

53. Sakuda M. Integrated information processing system in clinical orthodontics: An approach with use of a computer network system. Am J Orthod Dentofac Orthop. 1992;101:210-20.

54. Lowey MN. The development of a new method of cephalometric analysis with a computer-controlled, video image capture system, Part I: Br J Orthod. 1993;20(3):203-14.

55. Lowey MN. The development of a new method of cephalometric analysis with a computer-controlled, video image capture system, Part II: Br J Orthod.1993;20(4):313-31.

56. Prawat JS. A comparison between radiographic and sonically produced cephalometric values. Angle Orthod. 1995;65(4):271-6

57. Lim KF, Kelvin Weng CF. Phosphor stimulated computer cephalometry: Reliability of landmark identification. $\mathrm{Br} J$ Orthod. 1997;18:21-7.

58. Santoro M. Accuracy of digital and analogue cephalometric measurements assessed with the sandwich technique. Am J Orthod Dentofac Orthop. 2006;129:341-51.

59. Schulz RKW, Gloede MB. landmark identification on direct digital versus film based cephalometric radiographs: A human skull study. Am J Orthod Dent Orthop. 2002;122:635-42.

60. Jane. A comparison study of cephalometric measurements with digital versus manual methods $J$ Ind Orthod Soc. 2011;45(2):84-90

61. Chen YJ, Chen KS, Chang HF, Chen KC. Comparison of landmark identification in traditional versus computer-aided digital cephalometry. Angle Orthod. 2000;70(5):387-92

62. Baskin HN, Cineros GJ. A comparison of two computer cephalometric programs. J Clin Orthod. 1997;31(4):231-3.

63. Tumer PJ, Weerakone S. An evaluation of reproducibility of landmark identification using scanned cephalometric images. Br J Orthod. 2001;28:221-9.

64. Held CL, Ferguson DJ, Martin C. Cephalometric digitization: A determination of the minimum scanner settings necessary for precise landmark identification. Am J Orthod. 2001;119(5):472-81.

65. Johnson DR. Comparison of hand traced and computerized cephalograms: landmark identification, measurement and superimposition accuracy. Am J Orthod Dentofac Orthop. 2008;133:556-64.

66. Baurmrind S, Frantz RC. The reliablity of head film measurements. 1 landmark identification. Am J Orthod. 1971;60:111-27

67. Takeshi S, Savara BS. variability of cephalometrc landmarks used for face growth studies. Am J Orthod. 1972;61(6):603-18.

68. Gravely JF, and Murray Benzies P. Clinical significance of tracing error in cephalometry. Br J Orthod. 1974;1(3):95-101.

69. Trepkova B, Major P, Prasad N, Brian. N. Cephalometric landark identification and reproducibility: A Meta-Analysis. Am J Orthod Dentofac Orthop. 1997;112(2):165-70.
70. Ahlqvist J, Eliasson S, Welander U. The effect of projection errors on cephalometric length measurements. Eur J Orthod. 1986;8:141-8.

71. Houston WJB, Maher RE, Mc Elory D, Sherriff M. Sources of error in measurements from cephalometric radiographs. Eur $J$ Orthod. 1986;8:149-51.

72. Sayinsu K, Isik F, Trakyali G, Arun T. An evaluation of the errors in the cephalometric measurements on scanned cephalometric images and conventional tracings. Eur J Orthod 2007;29:105-8.

73. Nanda R, Nanda RS. Cephalometric study of the dentofacial complex. Angle Orthod. 1969;39:22-8.

74. Tikku T, Khanna R, Maurya RP, Srivastava K. Comparative evaluation of cephalometric measurements of monitor displayed images by Nemoceph software and its hard copy by manual Tracings. J Oral Biol Craniofac Res. 2014;4:35-41

75. Bruntz LQ. A comparison of scanned lateral cephalograms with corresponding original radiographs. Am J Orthod Dentofac. 2006;130(33):340-8.

76. Roden D, Johnson. Comparison of hand - traced and computerized cephalograms: Landmark identification, measurement, and superimposition accuracy. Am J Orthod. 2008;315-25.

77. Naoumova J, Lindman R. A comparison of manual traced images and corresponding scanned radiographs digitally traced. Eur J Ordhod. 2009;31(3):247-53.

78. Takada K. Automated Cephalometry: System Performace Reliability Using Landmark-Dependnet Criteria; Angle Orthod. 2009;79(6):1037-46

79. Tsorovas G, Karsten AL. A comparison of hand tracing and cephalometric analysis computer programs with and without advanced featutres- accuracy and time demands. Eur J Orthod. 2010;32(6):721-8

80. Guedes PA. A Comparative study of manual vs computerized cephalometric analysis. Dental Press J Orthod. 2010;15(2):4451

81. Erkan M. Reliability of computer generated ceophalometrics. Int J A Orthod Surg. 1995;(10);43-52.

82. Nadjmi N. Comparison of soft-tissue Profiles in Le Fort 1 osteotomy patients with Dolphin and Maxilim softwares. Am J Orthod Dentofacial Orthop. 2013;144:654-62.

83. Kumar D. Comparison of Manual and Computerized tracing using computer software: Burstone analysis: Int J Dental and Health Sci. 2014;1(2):131-40

84. Esteva Segura FJE. Comparative study between digital and manual cephalometry with digital radiographs. Revista Mexicana de orthodoncia' 2(2):93-96.

85. Sardana HK. Auto CEPH an Indigenous Software for 2-D Cephalometric Analysis. Am J Orthod Dentofac Orthop. 2008;35(2):812-40.

86. Oliver RG. Cephalometric analysis comparing five different methods. Br J Orthod 1991;18(4):277-83.

How to cite: Gaharwar JS. Computerised and manual cephalometry used for accuracy and reliability of landmark identification. Indian J Orthod Dentofacial Res. 2020;6(1):12-5. 